\title{
Inteligencia Emocional en el Deporte: Validación española del Schutte Self Report Inventory (SSRI) en deportistas espańoles
}

\author{
Emotional Inteligence in Sport: Validation of the Schutte \\ Self Report Inventory (SSRI) in Spanish athletes
}

\section{Inteligência emocional no esporte: validação espanhola do schutte self report inventory (SSRI) em esportistas espanhois}

\author{
Virginia García-Coll ${ }^{1}$, José-Luis Graupera-Sanz ${ }^{2}$, Luis-Miguel Ruiz-Pérez ${ }^{3}$ y Miriam Palomo-Nieto ${ }^{1}$
}

${ }^{1}$ Universidad de Castilla La Mancha, ${ }^{2}$ Universidad de Alcalá de Henares, ${ }^{3}$ Universidad Politécnica de Madrid

\begin{abstract}
Resumen: El propósito del estudio fue validar el Inventario de Inteligencia Emocional (SSRI) de Schutte et al. (1998) en una muestra de deportistas españoles de diferente nivel de pericia. Participaron 2091 deportistas (1519 hombres y 572 mujeres) de edades comprendidas entre los 11 y los 59 ańos $(M=20.8 ; D T=6.14)$. Los resultados de los AFE y AFC mostraron que el cuestionario presenta una estructura de cuatro dimensiones (percepción emocional, gestión auto-emocional, gestión hetero-emocional y utilización emocional), además de permitir obtener un valor de la escala general denominado Inteligencia Emocional en el Deporte. Las propiedades psicométricas y fiabilidad de la escala permiten presentar un inventario apto para la medición de la inteligencia emocional en el deporte.

Palabras clave: Competencia emocional, autopercepción, deporte, test.

Abstract: In this study we analyzed the validation and reliability of the SSRI Schutte's et al. (1998) Inventory of Emotional Intelligence using EFA and CFA with Spanish athletes of different expertise level. This sample was comprised of 2091 athletes (1519 males and 572 females) with a mean age of 20.8 years (ST: 6.14) and an age range of 11 to 59 years. The results obtained in this study presented a four dimensions structure (Emotional
\end{abstract}

perception, self-emotional management, hetero-emotional management and emotional utilization), and a global score of emotional intelligence in Sport. Psychometric properties and reliability of this instrument permits to offer an inventory apt to be applied in sport contexts.

Keywords: Emotional competence, sport, self-perception, test.

Resumo: O propósito desse estudo foi de validar o inventario de Inteligência Emocional (SSRI) de Schutte et al. (1998), em uma mostra de esportista espanhóis de diferentes níveis de habilidades. Participaram 2091 esportistas (1519 homens e 572 mulheres) de idades compreendidas entre 11 e 59 anos de idade $(\mathrm{M}=20.8$; DT= 6.14). Os resultados dos AFE e AFC mostraram que o questionário apresenta uma estrutura de quatro dimensóes (percepção emocional, gestão auto- emocional gestấo hetero - emocional e utilizaçấo emocional), ademais de permitir obter um valor de escala geral denominada Inteligência Emocional no Esporte. As propriedades psicométricas e fiabilidade da escala permitiram apresentar um inventario apto para medir a inteligência emocional de esportistas.

Palavras chave: Capacidade emocional, auto- percepção, esporte, test.

\section{Introducción}

Se acepta en la actualidad que las emociones, según diversos estudios (Côte y Miners, 2006; Pérez y Castejón, 2007; Prieto, Ferrándiz, Sánchez, y Bermejo, 2008; Zeidner, Matthews, y Roberts, 2004), influyen en diferentes ámbitos de la vida. Concretamente, las emociones juegan un papel importante en la vida de los deportistas y, directa o indirectamente, tienen influencia en aquello que quieren llevar a cabo (Hanin, 2000). Lo mismo ocurre con la inteligencia emocional, que a pesar de que su relación con el rendimiento deportivo no ha sido ampliamente estudiada, se ha convertido en un cons-

\footnotetext{
Dirección para correspondencia:

Virginia García-Coll

Virginia.Garcia@uclm.es

Agradecimientos: Este trabajo ha sido realizado con el apoyo de la Consejería de Educación y Ciencia de la Junta de Comunidades de Castilla La Mancha. Viceconsejería de Ciencia y Tecnología al primer autor.
}

tructo que recientemente está siendo estudiado con mucho interés en el contexto deportivo (Meyer y Fletcher, 2007).

Fue en 1990, cuando John Mayer y Peter Salovey definieron el concepto de inteligencia emocional y lo dotaron de modelo teórico (Mayer y Salovey, 1993) para explicar que algunas personas parecen ser más emocionalmente competentes que otras y la definieron como un tipo de inteligencia social que incluye la habilidad de supervisar y entender las emociones propias y las de los demás, discriminar entre ellas y usar la información para guiar el pensamiento y las acciones de uno mismo (189).

En un primer momento, este modelo constaba de tres categorías de habilidades adaptativas (Mayer y Salovey, 1993): valoración y expresión de la emoción (en sí mismo y en los demás), regulación de la emoción (tiene en cuenta los componentes de regulación de las emociones en los otros) y la utilización de las emociones para solucionar los problemas (incluye los compo- 
nentes de planificación flexible, pensamiento creativo, atención directa y la motivación). Más tarde el modelo se refina y se centra en que las emociones contienen información acerca de la conexión de las personas con otras personas y objetos, dando mayor énfasis a los componentes cognitivos y a la conceptualización de la inteligencia emocional (Mayer y Salovey, 1997).

A pesar de ello, el momento de mayor divulgación se produce con la publicación del best seller La Inteligencia Emocional del psicólogo y periodista Daniel Goleman en 1996. Como indicaba Goleman (1996), el grado de dominio que alcance una persona sobre la inteligencia emocional, resulta decisivo para determinar el motivo por el cual ciertas personas prosperaran en la vida, mientras que otros con un nivel de inteligencia similar acaban en un callejón sin salida. De esta manera, la inteligencia emocional (IE) ha supuesto un punto de inflexión en el estudio de las emociones, las cuales han pasado de ser elementos perturbadores de los procesos cognitivos a considerarse fenómenos vitales del ser humano que proporcionan información útil para solucionar problemas cotidianos (Extremera y Fernández-Berrocal, 2004).

Existen dos grandes teorías que se diferencian por la definición de inteligencia emocional y los instrumentos de medida empleados (Matthews, Zeidner, y Roberts, 2002). La primera utiliza las medidas de autoinforme y enfatiza la efectividad psicológica (éxito social, laboral o educativo), basándose en modelos de personalidad y ajustes no cognitivos (Bar-On, 1996; Goleman, 1998). Se utilizan cuestionarios, escalas e inventarios para que el participante dé una valoración subjetiva de sus niveles en ciertas habilidades y competencias afectivas. De esa forma se obtiene el índice de las creencias y expectativas de las personas sobre si pueden percibir, discriminar y regular sus emociones y las de los demás. Reciben el nombre de medidas de autoinforme y proporcionan una medida de inteligencia emocional percibida (Extremera, Fernández-Berrocal, Mestre, y Guil, 2004). En segundo lugar, aparece una nueva forma de evaluación más objetiva y que confía menos en la percepción de los propios participantes sobre sus habilidades emocionales. Están desarrolladas desde la perspectiva defendida por Mayer y Salovey. Su principal objetivo es medir la inteligencia emocional como una inteligencia clásica, tal como la inteligencia lógico- matemática o verbal de Howard Gardner (Gardner, 1983), es decir, mediante tareas de ejecución que el participante debe realizar supliendo así los problemas de sesgos que presentan los cuestionarios.

Está claro que el interés de la Psicología del Deporte por la inteligencia emocional cada vez va siendo mayor (Chan y Mallet, 2011; Crombie, Lombard y Noakes, 2009; Lane, Devonport, Soos, Karsai, Leibinger y Hamar, 2010; Lane, Thelwell, Lowther y Devonport, 2009 ; Meyer y Fletcher, 2007; Meyer y Zizzi, 2007; Thelwell, Lane, Weston, y Greenlees, 2008; Zizzi, Deaner, y Hirschhorn, 2003). Hasta la fecha son pocos los estudios que han examinado la inteligencia emocional en el deporte y nos han ofrecido resultados alentadores. Debido a ello es fundamental comprobar que las medidas de inteligencia emocional son válidas y fiables para su uso en el deporte. En este sentido, Lane, Meyer, Devonport, Davies, Thelwell, Gill, Diehl, Wilson, y Weston (2009), así como el grupo de investigación de Arruza y colaboradores (Arruza, Arribas, González, Balagué, Romero, y Ruiz, 2005; González, 2008) son los que han llevado a cabo este tipo de investigaciones utilizando el cuestionario Schutte Self Report Inventory (SSRI) (Schutte, Malouff, Hall, Haggerty, Cooper, Golden, y Dornhein, 1998) que tomó como partida el modelo original de inteligencia emocional de Salovey y Mayer (1990) así como el modelo revisado de dichos autores (Mayer y Salovey, 1997).

El SSRI es un instrumento que está compuesto por 33 ítems que evalúan como los sujetos son capaces de identificar, comprender y regular las emociones en sí mismo y en los otros, intentando abarcar de esta forma, las tres habilidades adaptativas del modelo inicial de Salovey y Mayer: valoración de las emociones en uno mismo y en los otros, regulación de las emociones en si mismo y en los otros y utilización de las emociones de cara a resolver problemas. Existe una controversia respecto a la estructura factorial del instrumento ya que se pasó de pensar que era una escala multidimensional a, posteriormente, decir que era unidimensional. Así, Schutte et al. (1998) encontraron un único factor general, mientras que Chico (1999), Austin, Saklofshe, Huang, y McKenney (2004) y Ferrándiz, Martín, Gallud, Ferrando, López-Pina, y Prieto (2006) encontraron que estaba formado por tres factores diferentes. Mientras que Petrides y Furnham (2000), Ciarrochi, Deane, y Anderson (2002) y Arruza et al. (2005) proponen una solución tetrafactorial, y Lane et al. (2009) una solución de seis factores.

En general estos hallazgos confirman que dicha escala es una medida de inteligencia emocional de autoinforme con claras evidencias de fiabilidad, adecuada validez con un carácter distintivo respecto de otras variables de personalidad sólidamente constatadas y con una demostrada capacidad predictiva. A pesar de todo, estudios dirigidos a comprobar exclusivamente la estructura factorial de la escala constatan debilidades que no fueron concebidas por sus creadores. En primer lugar, algunos estudios (Ferrándiz et al., 2006; Ciarrochi, Deane, y Anderson, 2002; Lane et al., 2009) han encontrado que la estructura factorial de la escala no se ajusta a un único factor general de inteligencia emocional tal como propusieron sus autores originalmente. En segundo lugar, la escala presenta problemas de aquiescencia debido a la escasez de ítems en sentido negativo y en tercer lugar, su estructura no se ajusta exactamente a la teoría del modelo original de Salovey y Mayer formado por tres componentes ya que, como hemos dicho anteriormente, Schutte et al. (1998) encontraron un solo factor mientras que otros estudios posteriores encontraron 4 (Petrides y Furnham, 2000; Saklofske, Austin, y Minski, 2003). Actualmente, existe una versión mejorada de la escala en inglés compuesta por 41 ítems que comprende tres dimensiones (Austin, Saklofke, Huangh, 
y McKenney, 2004): evaluación emocional, utilización de las emociones y regulación emocional.

En el ámbito deportivo, Arruza et al. (2005) desarrollaron La Escala de Competencia Emocional con el objetivo de evaluar en los deportistas las emociones que, como ya sabemos, juegan un papel importante dentro de los contextos deportivos. Estos autores analizaron los antecedentes y las características psicométricas de la Escala de Desarrollo Emocional de Schutte et al. (1998), ya validado en el campo de la psicología general y para adaptarlo, se eligió la versión traducida al español realizada por Eliseo Chico (1999).

Recientemente han sido González (2008) y Lane et al. (2009) los que han adaptado y validado este instrumento dentro del ámbito deportivo. Hasta ahora al correlacionar medidas de inteligencia emocional con parámetros de rendimiento atlético se recurría a cuestionarios ya validados (herramientas empleadas habitualmente en el ámbito laboral y de los recursos humanos) pero que nada tenía que ver con el contexto deportivo. Estos investigadores han tratado de desarrollar un instrumento que mida el nivel de inteligencia emocional de los deportistas, específico para este contexto. González (2008) llegó a un instrumento de autoinforme que, a pesar de no mostrar todavía unos índices de validez y fiabilidad satisfactorios, constituye un buen punto de partida de cara a construir una escala que mida la inteligencia emocional desde el modelo de Salovey y Mayer en el contexto competitivo. Lane et al. (2009) evaluaron la validez factorial del instrumento con un amplio número de deportistas, obteniendo resultados alentadores con una versión reducida de 19 ítems que se agrupaban en una estructura factorial de 6 factores diferentes. A pesar de ello, ambos estudios recomiendan seguir en esta línea de investigación para poder comprobar la verdadera validez del instrumento.

Los problemas que aparecen en todas las investigaciones tienen que ver con la definición del constructo y su evaluación, ya que no queda clara la estructura factorial inherente. Serían necesarios estudios con mayor tamańo muestral para confirmar tal estructura (Ferrandiz et al., 2006; Arruza et al., 2005, ). Por lo tanto, se hacía necesario el análisis de las propiedades psicométricas de este instrumento. De ahí que los objetivos de evaluación psicométrica planteados fueran adaptar al contexto deportivo y validar en población deportiva española el Inventario de Inteligencia Emocional (IE) de Schutte et al. (1998), estableciendo su estructura factorial y analizando la invarianza métrica y conceptual en función del sexo, así como analizar la fiabilidad de las puntuaciones del instrumento mediante la consistencia interna y el test- retest.

\section{Método}

\section{Participantes}

En esta investigación participaron voluntariamente 2091 deportistas (1519 varones y 572 mujeres) de edades comprendidas entre los 11 y los 59 años $(M=20.8 ; D T=6.14)$. El nivel de competición se estableció en tres niveles diferentes: autonómico $(\mathrm{n}=793)$, nacional $(\mathrm{n}=919)$ e internacional $(\mathrm{n}=365)$ Todos los participantes fueron informados de la experiencia y firmaron su consentimiento. Además, se empleó una muestra complementaria de 67 deportistas, a la que se le aplicó el cuestionario en dos ocasiones, con una separación de 15 días entre ambas, al efecto de calcular la estabilidad (test-retest) del mismo.

\section{Instrumento}

Se empleó el Inventario de Inteligencia Emocional de Schutte et al. (1998). Este instrumento fue desarrollado para población general y su validación ofreció una solución unifactorial, con un conjunto de 33 ítems, una consistencia interna de .87, y una fiabilidad-estabilidad de .78. La valoración se llevó a cabo en una escala tipo Likert de 5 puntos, siendo el 1 Totalmente en desacuerdo y el 5 Totalmente de acuerdo.

\section{Procedimiento}

El procedimiento seguido para la administración del instrumento a los deportistas consistió, en primer lugar, en contactar directamente con los responsables de los equipos deportivos para darles a conocer el estudio y solicitar su permiso. Posteriormente se contactó con los deportistas quienes firmaron un documento en el que consentía su participación en el estudio. A los menores de edad se les solicitó una autorización paterna. Los cuestionarios fueron aplicados en los lugares de entrenamiento de los diferentes equipos. El tiempo para cumplimentarlo osciló entre diez y quince minutos, no presentando en su mayoría dificultades de ningún tipo para su comprensión por parte de los jugadores.

\section{Análisis de los Datos}

Para establecer la validez de constructo de este cuestionario, dado que sobre él se han formulado distintos modelos estructurales, se siguió un procedimiento analítico secuencial. En primer lugar, se realizó un análisis factorial exploratorio para establecer los modelos dimensionales hipotéticos de inteligencia emocional. En segundo lugar se pusieron a prueba estos modelos mediante la aplicación de análisis factoriales confirmatorios. En tercer lugar se seleccionó el modelo más ajustado. Finalmente se contrastaron los supuestos de invarianza de la configuración y de invarianza métrica entre las muestras de hombres y mujeres. Para los análisis estadísticos se empleó el programa SPSS 18 y, específicamente para los análisis factoriales confirmatorios, el método de máxima verosimilitud con el procedimiento robusto de Satorra y Bentler implementado en el programa EQS 6.1. 


\section{Resultados}

\section{Análisis de propiedades psicométricas}

Para llegar a esta solución fue preciso eliminar los ítems 5, 28 y 33 que estaban redactados en forma negativa y que formaban una especie de factor de respuesta que perturbaba el conjunto del análisis. Una vez extraídos los cuatro factores se aplicó una rotación oblicua de tipo promax, obteniéndose coeficientes de correlación considerables (superiores a .30) en todos los casos.

\section{Análisis factorial exploratorio (AFE)}

Como paso previo al AFE se comprobó si la matriz de correlaciones entre los ítems cumplía las condiciones de aplicación adecuadas para este tipo de análisis. En la prueba de esfericidad de Bartlett se obtuvo un valor alto y significativo $\left(\chi^{2}(435)=16347.78 ; p<.001\right)$, por lo que se consideró que la matriz de correlaciones entre los ítems no era esférica y resultaba adecuada para la factorización. La medida de adecuación muestral $(M S A=.95)$ obtenida mediante el índice global de Kaiser, Meyer, y Olkin fue superior a .90, por lo que la matriz pudo considerarse excelente para la aplicación del análisis factorial. Se aplicó el procedimiento de análisis de componentes principales, obteniéndose cuatro factores principales (con autovalores superiores a 1) que explicaron un $42 \%$ de la varianza $(27.79 \%, 5.84 \%$, $4.05 \%$ Y $4.01 \%$ respectivamente).

Análisis factorial confirmatorio (AFC) del modelo unifactorial

Los resultados del AFE, por una parte, mostraron un modelo tetrafactorial con factores relacionados. Es decir, que se obtuvo un modelo similar al propuesto por Petrides y Furnham (2000) y Ciarrochi, Deane y Anderson (2002). Pero, por otra parte, los resultados no fueron claramente incompatibles con la hipótesis de un modelo unifactorial, puesto que el primer factor explicó un porcentaje de varianza muy elevado, mayor del 20\%, y muy superior al del segundo factor. Con resultados muy semejantes los constructores del test establecieron un modelo unifactorial (Schutte et al., 1998). En consecuencia se procedió a realizar sendos AFC para contrastar cuál de los dos modelos presentaba un mejor ajuste.

Planteamiento del modelo hipotético unifactorial. Se consideró que la inteligencia emocional en el deporte era un constructo unidimensional. Por ello se configuró un factor que incluía los 30 ítems del test (recuérdese que se eliminaron los tres redactados en forma negativa). Con ello se estableció un modelo unifactorial hipotético de inteligencia emocional en el deporte empleando el diseño formal y la nomenclatura habituales en los métodos de ecuaciones estructurales y de análisis factorial confirmatorio, teniendo en cuenta tanto los resultados del AFE, como el planteamiento unidimensional de Schutte et al. (1998).

Análisis de las condiciones de aplicación del AFC: normalidad multivariante. Los coeficientes univariantes de asimetría y curtosis se encuentran entre -2 y 2 en todos los ítems, por lo que puede considerarse que sus distribuciones no se alejan demasiado de la normal (Pérez, 2004) (Tabla 1). En cuanto a la normalidad multivariante el coeficiente de curtosis de Mardia indica una distribución que se aleja significativamente de la normal-multivariante. Conviene tener en cuenta que el gran número de variables y, sobre todo, el gran tamańo de la muestra hacen que este tipo de pruebas estadísticas sean demasiado sensibles a desviaciones de la normalidad que pueden ser muy ligeras.

Tabla 1. Coeficientes de asimetría y curtosis de los ítems y coeficiente de Mardia de kurtosis multivariante.

\begin{tabular}{|c|c|c|c|c|}
\hline Ítem & Media & DT & Asimetría & Curtosis \\
\hline IED01 & 3.83 & 1.08 & -.72 & -.11 \\
\hline IED02 & 3.86 & .93 & -.63 & .10 \\
\hline IED03 & 4.39 & .76 & -1.20 & 1.30 \\
\hline IED04 & 3.91 & .78 & -.30 & -.58 \\
\hline IED06 & 3.89 & .90 & -.53 & -.29 \\
\hline IED07 & 3.73 & 1.00 & -.64 & .10 \\
\hline IED08 & 4.14 & .88 & -.81 & .12 \\
\hline IED09 & 4.01 & .80 & -.57 & -.01 \\
\hline IED10 & 4.48 & .79 & -1.57 & 1.89 \\
\hline IED11 & 3.91 & 1.04 & -.74 & -.13 \\
\hline IED12 & 3.67 & .90 & -.35 & -.14 \\
\hline IED13 & 3.79 & .81 & -.41 & -.07 \\
\hline IED14 & 4.27 & .72 & -.88 & .56 \\
\hline IED15 & 3.88 & .84 & -.47 & -.14 \\
\hline IED16 & 3.90 & .90 & -.69 & .12 \\
\hline IED17 & 4.28 & .82 & -1.10 & 1.16 \\
\hline IED18 & 3.80 & .92 & -.54 & -.02 \\
\hline IED19 & 3.62 & 1.06 & -.48 & -.31 \\
\hline IED20 & 4.20 & .83 & -.95 & .76 \\
\hline IED21 & 3.65 & .95 & -.46 & -.04 \\
\hline IED22 & 3.73 & .87 & -.40 & .09 \\
\hline IED23 & 4.16 & .88 & -.88 & .36 \\
\hline IED24 & 4.47 & .77 & -1.42 & 1.69 \\
\hline IED25 & 3.89 & .89 & -.57 & .07 \\
\hline IED26 & 3.56 & .92 & -.37 & -.21 \\
\hline IED27 & 3.53 & .91 & -.28 & -.09 \\
\hline IED29 & 3.46 & .92 & -.20 & -.20 \\
\hline
\end{tabular}




\begin{tabular}{lcccc} 
IED30 & 4.25 & .81 & -1.00 & .96 \\
IED31 & 3.99 & .86 & -.70 & .37 \\
IED32 & 3.66 & .97 & -.43 & -.10 \\
\hline Curtosis multivariante & \multicolumn{2}{l}{ Coeficiente de Mardia } & 204.62 \\
& & Razón crítica & 106.77 \\
& & p & $<.001$ \\
\hline
\end{tabular}

En cualquier caso, para prevenir el posible efecto de la falta de normalidad multivariante se elige como método de estimación del modelo factorial el de máxima verosimilitud con el procedimiento robusto de Satorra y Bentler para el cálculo de los estadísticos de bondad de ajuste y los errores típicos. Como se ha mencionado ya en páginas anteriores, este modus operandi es el recomendado para el caso de distribuciones que carezcan de normalidad multivariante en grandes muestras (Byrne, 2006).

En cuanto a los pesos o coeficientes de regresión estimados cabe decir que todos resultaron ser significativos $(p<.001)$. Una vez estandarizados (Figura 1) pudo comprobarse que la mayoría de ellos (27) eran iguales o superiores a .40 (16 de ellos mayores de .50$)$, el resto obtuvieron coeficientes superiores a .35. No se observaron signos de multicolinealidad (factor de inflación de varianza VIFmax=1.66 <5; número de condicionamiento $\kappa(\mathrm{R})=1.34<10$, los errores típicos de los regresores fueran pequeños y similares en todos los factores).

Figura 1. Modelo métrico unifactorial de inteligencia emocional en el deporte (coeficientes estandarizados).

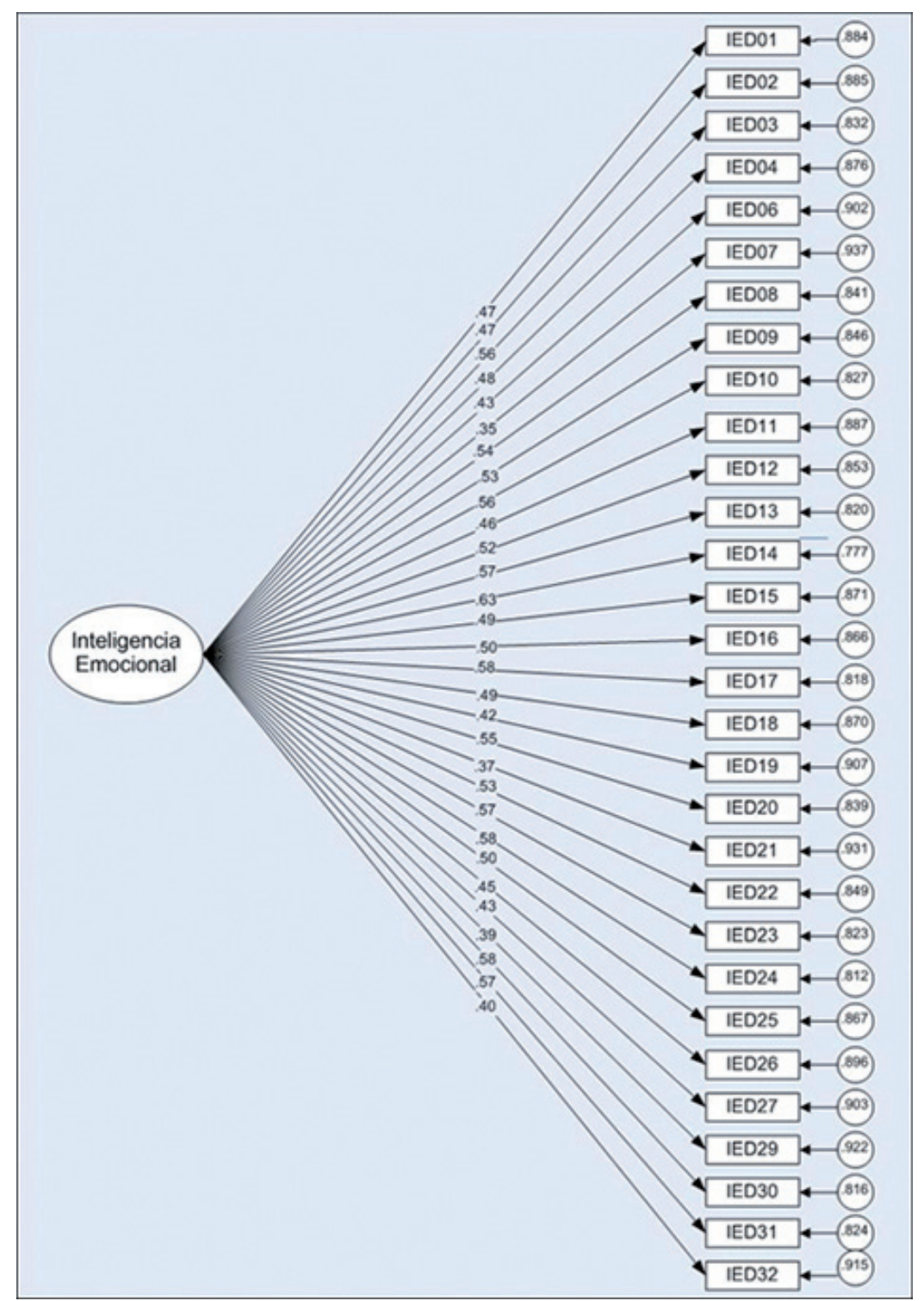


Los índices de ajuste seleccionados, siguiendo la recomendación de Marsh (2007), fueron los siguientes: índice de ajuste no normado $(\mathrm{NNFI}=.84)$, índice de ajuste comparativo $(\mathrm{CFI}=.85)$ y raíz cuadrática media del error de aproximación estandarizado (RMSEA= .049). El índice RMSEA inferior a .05 fue un indicador de un ajuste aceptable; pero los índices NNFI y CFI inferiores a .90 manifestaron un ajuste pobre.

\section{Análisis factorial confirmatorio (AFC) del modelo tetrafactorial}

Planteamiento del modelo hipotético. Los resultados del AFE presentado en el apartado anterior mostraron un modelo tetrafactorial con factores relacionados. El modelo estructural de inteligencia emocional que se obtuvo fue similar al propuesto por Petrides y Furnham (2000) y Ciarrochi, Deane y Anderson (2002). Se consideró hipotéticamente que la inteligencia emocional en el deporte estaba configurada por los siguientes factores, siguiendo la denominación propuesta por los últimos autores citados: percepción emocional (ocho ítems), gestión auto-emocional (ocho ítems), gestión heteroemocional (diez ítems) y utilización emocional (cuatro ítems).

Estimación del modelo métrico tetrafactorial. Se eligió la aplicación del método de máxima verosimilitud para la estimación de los parámetros del modelo con el procedimiento robusto de Satorra y Bentler para el cálculo de los estadísticos de bondad de ajuste y los errores típicos. En cuanto a los pesos o coeficientes de regresión estimados cabe decir que todos resultaron ser significativos $(p<.001)$. Una vez estandarizados pudo comprobarse que la mayoría de ellos (22) eran iguales o superiores a .50 , el resto obtuvieron coeficientes superiores a .40, excepto el ítem 21 con un valor ligeramente inferior (.36) (Figura 1). Las covarianzas interfactoriales también resultaron ser significativas $(\mathrm{p}<.001)$. Una vez estandarizadas se obtuvo que las seis intercorrelaciones fueron superiores a 70. Las mayores se produjeron entre la percepción emocional y otros dos factores: la gestión hetero-emocional (.93) y la utilización emocional (.87). El resto de las intercorrelaciones oscilaron entre .70 y .80 . No se observaron signos de multicolinealidad (factor de inflación de varianza VIFmáx= $1.77<$ 5 ; número de condicionamiento $\kappa(\mathrm{R})=1.36<10$, los errores típicos de los regresores fueron pequeños y similares en todos los factores).

El índice de ajuste no normado (NNFI= 89$)$, índice de ajuste comparativo $(\mathrm{CFI}=.90)$ y raíz cuadrática media del error de aproximación estandarizado (RMSEA = .041). Dado que los índices NNFI y CFI fueron iguales a .90 y el RMSEA menor que .05 el ajuste se consideró aceptable (Marsh, 2007) (Tabla 2).
Tabla 2. Índices de bondad de ajuste del modelo métrico tetrafactorial.

\begin{tabular}{lrcc}
\hline \multirow{2}{*}{$\begin{array}{l}\text { Índices de bondad } \\
\text { de ajuste }\end{array}$} & \multirow{2}{*}{ Estimación } & \multicolumn{2}{c}{ Criterios de ajuste* } \\
\cline { 3 - 4 } & & Aceptable & Excelente \\
\hline NNFI & .89 & $\geq .90$ & $\geq .95$ \\
CFI & .90 & $\geq .90$ & $\geq .95$ \\
RMSEA & .041 & $\leq .08$ & $\leq .05$ \\
IC (90\%) & $.039-.043$ & & \\
\hline * Criterios de bondad de ajuste basados en Marsh (2007, p. 785)
\end{tabular}

En los apartados anteriores se ha señalado que el modelo tetrafactorial obtuvo un buen ajuste, mientras que el unifactorial obtuvo un ajuste más pobre. Ahora bien, al tratarse de modelos no anidados (con un diferente número de parámetros libres estimados) se consideró conveniente calcular índices comparativos de ajuste. Todos estos índices (AIC, CAIC y ECVI) resultaron sensiblemente menores en el modelo tetrafactorial, por ello se eligió este modelo como el más ajustado a la configuración latente de la inteligencia emocional en el deporte (Tabla 3).

\section{Estabilidad temporal}

El proceso de validez de constructo, desarrollado en el epígrafe anterior, concluyó que el test aquí analizado medía cuatro factores de la inteligencia emocional en el deporte. Por ello se procedió a establecer una estructura de puntuación del test constituida por cuatro escalas sumativas, correspondientes a los factores obtenidos en el AFC (percepción emocional, gestión auto-emocional, gestión hetero-emocional y utilización emocional). Además se decidió, dado que los cuatro factores antedichos están muy relacionados positivamente entre sí, calcular una escala general (inteligencia emocional). El cálculo de las puntuaciones de cada una de estas escalas se realizó mediante el promedio de los ítems que las constituyen.

Tabla 3. Índices comparativos de los modelos unifactorial y tetrafactorial.

\begin{tabular}{lrc}
\hline Índices comparativos & \multicolumn{1}{l}{ Modelo } \\
\cline { 2 - 3 } de bondad de ajuste & Unifactorial & Tetrafactorial \\
\hline S-B $\chi^{2}(435)$ & 2413.37 & 1781.80 \\
Parámetros libres & 60 & 66 \\
AIC & 1603.37 & 983.80 \\
CAIC & 2932.09 & 2352.40 \\
ECVI & 1.21 & 0.92 \\
\hline
\end{tabular}


Figura 2. Modelo métrico tetrafactorial de inteligencia emocional en el deporte (coeficientes estandarizados).

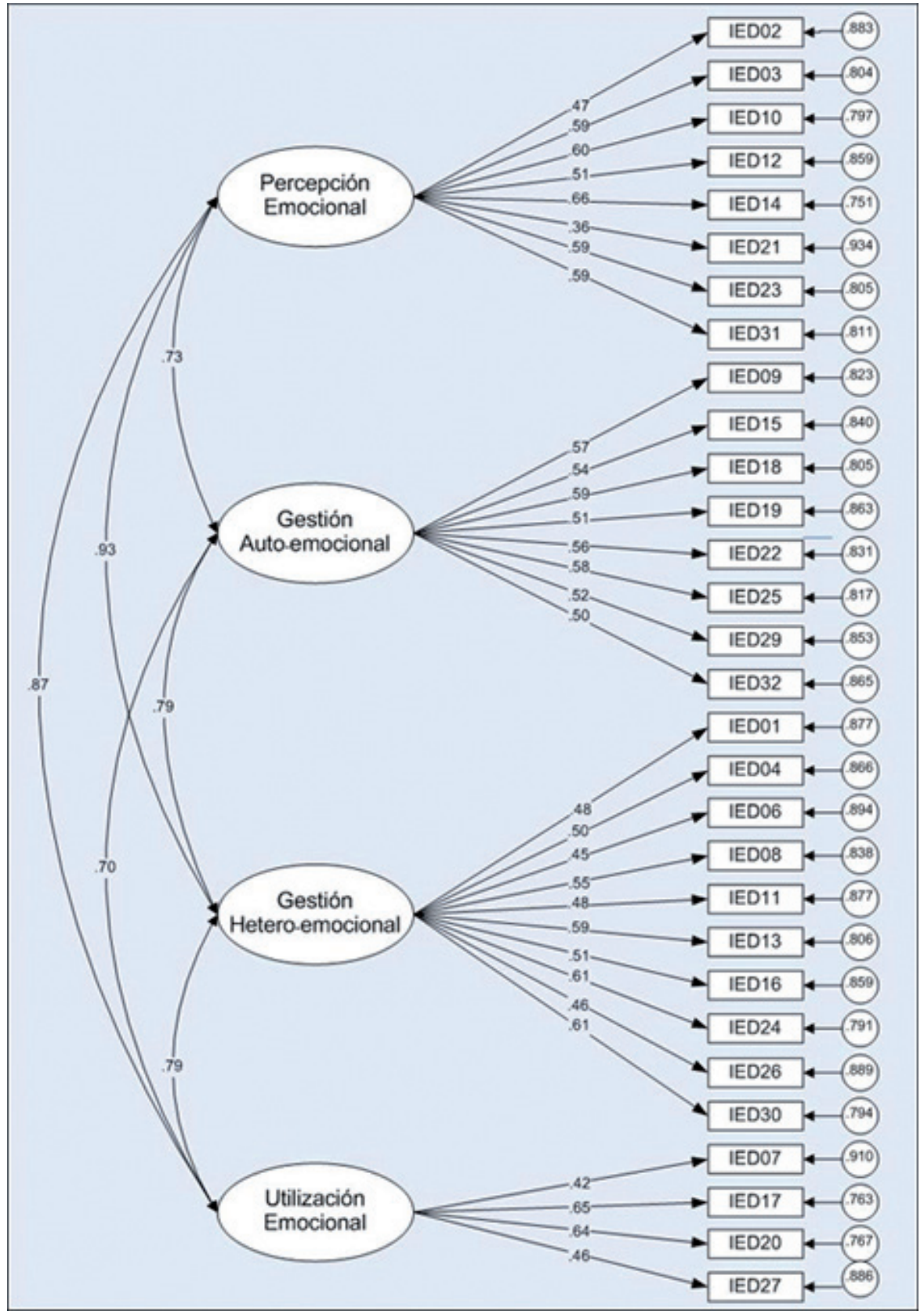

Para estimar la fiabilidad de las escalas se emplearon dos procedimientos complementarios: consistencia interna, mediante el coeficiente $\alpha$ de Cronbach, y test-retest. Los coeficientes $\alpha$ de las escalas calculados con la muestra total fueron los siguientes: en la subescala de percepción emocional .77, en gestión auto-emocional .77, en gestión hetero-emocional .78 y en utilización emocional .63. Excepto esta última escala, los coeficientes fueron significativamente superiores a .70 (Nunnally, 1978).
En cuanto a la escala general de inteligencia emocional, que cuenta con un número de ítems mayor, el coeficiente $\alpha$ obtenido mediante el procedimiento de fiabilidad compuesta obtuvo un valor de .91 que se considera adecuado incluso para tomar decisiones diagnósticas en Psicología del Deporte (DeVellis, 2003; Graupera, 2007; Netemeyer, Bearden y Sharma, 2003). 
Tabla 4. Fiabilidad de las escalas.

\begin{tabular}{lccccc}
\hline \multirow{2}{*}{ Escala } & Número & Test-retest & $\alpha$ & \multicolumn{2}{c}{ Intervalo de confianza de $\alpha(95 \%)$} \\
\cline { 6 - 6 } & de ítems & $(\mathrm{n}=67)$ & $(\mathrm{n}=2091)$ & Límite inferior & Límite superior \\
\hline Percepción emocional & 8 & .51 & .77 & .75 & .78 \\
Gestión auto-emocional & 8 & .71 & .77 & .76 & .79 \\
Gestión hetero-emocional & 10 & .36 & .78 & .77 & .80 \\
Utilización emocional & 4 & .32 & .63 & .60 & .65 \\
Inteligencia emocional & 30 & .69 & $.91^{*}$ & .90 & .91 \\
\hline
\end{tabular}

${ }^{*}$ Coeficiente obtenido mediante el procedimiento de fiabilidad compuesta.

Como indicadores de la estabilidad temporal de las puntuaciones de las distintas escalas se calcularon coeficientes de fiabilidad test-retest. En este caso se contó con 67 deportistas que cumplimentaron el test de manera repetida, con un intervalo de quince días entre las aplicaciones. Los coeficientes obtenidos con las escalas parciales fueron moderados o bajos (entre .32 y .71) y el correspondiente al test completo alcanzó un valor de .69 (Tabla 4).

\section{Análisis de la invarianza por sexo}

Para comprobar la invarianza factorial del modelo de inteligencia emocional en el deporte en los hombres y las mujeres, se evaluó la invarianza de configuración en primer lugar y después la invarianza métrica. En cuanto a los pesos o coeficientes de regresión cabe decir que todos resultaron ser significativos $(\mathrm{p}<.001)$ en ambos sexos. Una vez estandarizados pudo comprobarse que casi todos ellos eran iguales o superiores a .50 ( 21 en las mujeres y 22 en los hombres) o a .40 ( 7 en ambos grupos). Las excepciones, que obtuvieron unos valores próximos pero algo inferiores a .40 , fueron el ítem 21 en los dos grupos (.39 en las mujeres y .35 en los hombres) y el ítem 7 en las mujeres (.39). Las covarianzas interfactoriales ( $\psi \mathrm{ij}$, Tabla 5) también resultaron ser significativas ( $\mathrm{p}<.001)$. Una vez estandarizadas se obtuvo que las seis intercorrelaciones fueron elevadas, entre .70 y .93 , en ambos grupos.

Tabla 5. Covarianzas interfactoriales ( $\psi$ ij) del modelo métrico en mujeres y hombres.

\begin{tabular}{|c|c|c|c|c|c|c|}
\hline \multirow[b]{2}{*}{ Parámetro } & \multicolumn{3}{|c|}{ Mujeres } & \multicolumn{3}{|c|}{ Hombres } \\
\hline & Covarianza & Error típico & Razón crítica & Covarianza & Error típico & Razón crítica \\
\hline$\psi \mathrm{ij} 1,2$ & .15 & .018 & $8.09^{*}$ & .20 & .014 & $14.12^{*}$ \\
\hline$\psi \mathrm{ij} 1,3$ & .18 & .023 & $7.84^{*}$ & .24 & .017 & $13.89^{*}$ \\
\hline$\psi \mathrm{ij} 1,4$ & .19 & .021 & $8.91^{*}$ & .23 & .015 & $15.43^{*}$ \\
\hline$\psi \mathrm{ij} 2,3$ & .20 & .025 & $7.84^{*}$ & .21 & .017 & $12.78^{*}$ \\
\hline$\psi \mathrm{ij} 2,4$ & .19 & .022 & $8.27^{*}$ & .21 & .015 & $13.56^{*}$ \\
\hline$\psi \mathrm{ij} 3,4$ & .21 & .026 & $8.12^{*}$ & .21 & .016 & $13.03^{*}$ \\
\hline
\end{tabular}

${ }^{*} \mathrm{p}<.001$.

Se calcularon, en ambos grupos, los mismos índices de ajuste que en el caso del análisis de la muestra general. El índice de ajuste no normado (NNFI) fue de .86 en las mujeres y .89 en los hombres. El índice de ajuste comparativo (CFI) alcanzó valores de .87 en las mujeres y .90 en los hombres. En el caso de la raíz cuadrática media del error de aproximación estandarizado (RMSEA) se obtuvieron valores de .044 y .041 respectivamente. En las mujeres el ajuste pudo considerarse aceptable teniendo en cuenta el RMSEA menor que .05; aunque el índice CFI, algo menor que .90, solo se aproximó a la aceptabilidad. En los hombres el ajuste pudo considerarse globalmente bueno (RMSEA bastante menor que .05 e índice CFI igual a .90).
Bondad de ajuste del AFC multigrupo. Una vez comprobado que el ajuste del modelo hipotético de Inteligencia emocional en el deporte era aceptable en los dos sexos, analizados separadamente, se procedió a analizar la invarianza de configuración mediante un AFC multigrupo sin restricciones. Los índices de ajuste se calcularon mediante la estimación reescalada de $\chi^{2}\left(\mathrm{~S}-\mathrm{B} \chi^{2}(798)=2186.92 ; \mathrm{p}<.001\right)$. El índice de ajuste no normado (NNFI) fue de .89 , el índice de ajuste comparativo (CFI) fue de 90 y la raíz cuadrática media del error de aproximación estandarizado (RMSEA) fue .029. Dado que los índices NNFI y CFI fueron muy próximos o iguales a .90 y el RMSEA muy inferior a .05, el ajuste de configuración pudo considerarse aceptable (Tabla 6). 
Análisis de la invarianza métrica. Una vez comprobada la invarianza de configuración del modelo de Inteligencia emocional en el deporte en los dos sexos, se procedió a la evaluación de la invarianza métrica. Para ello se llevó a cabo un AFC multigrupo (hombres y mujeres) con las siguientes restricciones: igualdad de los coeficientes de regresión, e igualdad de las covarianzas interfactoriales. La estimación reescalada de $\chi^{2}$ mediante el procedimiento robusto de Satorra y Bentler fue: S-B $\left.\chi^{2}(830)=2330.83 ; \mathrm{p}<.001\right)$. Los índices de ajuste no normado (NNFI) y el índice de ajuste comparativo (CFI) alcanzaron valores próximos a .90 (.88 y .89 respectivamente) y la raíz cuadrática media del error de aproximación estandarizado (RMSEA) fue .029. Dado que los índices NNFI y CFI se aproximaron a $.90 \mathrm{y}$ el RMSEA fue muy inferior a .05 , el ajuste métrico del AFC multigrupo con restricciones pudo considerarse aceptable (Tabla 6).

Tabla 6. Índices de bondad de ajuste de los AFC multigrupo sin restricciones y con restricciones.

\begin{tabular}{|c|c|c|c|c|}
\hline \multirow{2}{*}{$\begin{array}{l}\text { Índices de bondad } \\
\text { de ajuste }\end{array}$} & \multirow{2}{*}{$\begin{array}{l}\text { Invarianza de configuración } \\
\text { (AFC sin restricciones) }\end{array}$} & \multirow{2}{*}{$\begin{array}{c}\text { Invarianza métrica } \\
\text { (AFC con restricciones) }\end{array}$} & \multicolumn{2}{|c|}{ Criterios de ajuste* } \\
\hline & & & Aceptable & Excelente \\
\hline NNFI & .89 & .88 & $\geq .90$ & $\geq .95$ \\
\hline CFI & .90 & .89 & $\geq .90$ & $\geq .95$ \\
\hline RMSEA & .029 & .029 & $\leq .08$ & $\leq .05$ \\
\hline IC (90\%) & $.027-.030$ & $.028-.031$ & & \\
\hline
\end{tabular}

* Criterios de bondad de ajuste basados en Marsh (2007, p. 785)

Si se comparan los índices de bondad de ajuste que se obtuvieron en el AFC sin restricciones y en el AFC con restricciones (ambos en la Tabla 6) puede observarse que son muy similares, por lo que no se aprecia una degradación significativa en el modelo con restricciones. En consecuencia, en el modelo estructural de Inteligencia emocional en el deporte se obtuvo un conjunto de relaciones (saturaciones, $\lambda$ ij y covarianzas, $\psi \mathrm{ij}$ ) muy similar en los dos sexos.

\section{Discusión}

El objetivo de este estudio fue adaptar y validar al contexto español la Escala de Inteligencia Emocional de Schutte et al. (1998) con ligeras adaptaciones al ámbito deportivo. Este instrumento fue desarrollado para la población en general y no para el deporte, y no está exento de controversia en cuanto a su modelo estructural, pasando de la solución unifactorial de los autores originales, a otras polidimensionales (Chico, 1999; Ciarrochi, Deane y Anderson, 2002; Lane et al., 2009; Petrides y Furham, 2000).

El desacuerdo encontrado entre los distintos trabajos, y el hecho de adaptarlo al ámbito del deporte, hizo necesario llevar a cabo un proceso inicial de valoración del constructo estudiado, para lo cual se requería un análisis con un mayor tamaño muestral para confirmar tal estructura (Ferrándiz et al., 2000), lo cual se ha convertido en una de las aportaciones originales de este trabajo. En estudios anteriores Arruza et al. (2005) habían hecho lo mismo basándose en la traducción al español que hizo Eliseo Chico (1999) fuera del ámbito deportivo, planteando la posibilidad de incluir mejoras sustanciales al cuestionario para futuras versiones relacionadas con su aplicabilidad.

Mediante un análisis exploratorio se establecieron dos modelos dimensionales hipotéticos de inteligencia emocional, que se pusieron a prueba mediante la aplicación de análisis factoriales confirmatorios, para seleccionar el modelo más ajustado. Por un lado, un modelo tetrafactorial con factores relacionados similar al propuesto por Petrides y Furham (2000) y Ciarrochi, Deane, y Anderson (2002), y por otra, un modelo unifactorial con resultados muy similares a los obtenidos por los creadores del test.

Tras el análisis factorial confirmatorio se llegó a la conclusión de que el modelo tetrafactorial era el más ajustado a la configuración latente de la inteligencia emocional en el deporte. Siguiendo la denominación de Ciarrochi, Deane, y Anderson (2002), se consideró que la inteligencia emocional en el Deporte estaba configurada por los factores: percepción emocional, gestión auto-emocional, gestión hetero-emocional y utilización emocional. Además se decidió calcular una escala general que se denominó inteligencia emocional. Estos resultados no coinciden con el último estudio de validación realizado de este instrumento por Lane et al. (2009).

El siguiente paso fue analizar la invarianza conceptual y métrica del instrumento en función del sexo, donde se obtuvo un conjunto de relaciones muy similar en los dos sexos y, finalmente, se analizó la fiabilidad en las distintas escalas a través del coeficiente alpha de Cronbach y el test-retest. Los coeficientes $\alpha$ de las subescalas calculadas con la muestra, a excepción de la escala utilización emocional que fue de .63, fueron superiores a .70, llegando de esta forma al límite de aceptabilidad de los coeficientes de fiabilidad. En cuanto a la escala general de inteligencia emocional se obtuvo un valor de .91, que se considera muy adecuado incluso para tomar decisiones diagnósticas en Psicología del Deporte (DeVellis, 2003; Graupera, 2007; Netemeyer, Bearden y Sharma, 2003). Los coeficientes de fiabilidad test- retest también fueron mo- 
derados o bajos (entre .32 y .71) y el correspondiente al test completo alcanzó un valor de .69 .

Como conclusión podemos decir que el test de inteligencia emocional, adaptado al deporte quedó configurado como un modelo tetradimensional, con los siguientes factores: percepción emocional, gestión auto-emocional, gestión heteroemocional y utilización emocional. Este modelo, similar al propuesto por Petrides y Furnham (2000) y Ciarrochi, Deane, y Anderson (2002), quedó confirmado con una bondad de ajuste aceptable y mostró invarianza métrica y de configuración en mujeres y hombres. La escala total del test, denominada inteligencia emocional, obtuvo un coeficiente de consistencia interna elevado, por lo que el test resultó adecuado para su aplicación general en deportistas e, incluso, para tomar decisiones diagnósticas en Psicología del Deporte. Las cuatro escalas parciales presentaron una consistencia interna aceptable.

Sin duda una de las aspiraciones que las Ciencias del Deporte, y en concreto la Psicología del Deporte tienen, es el desarrollo de instrumentos que evalúen de forma específica las demandas de los propios deportes. Es clásica la tendencia a adaptar cuestionarios o inventarios que fueron desarrollados para otros contextos, al deporte. En el caso de la inteligencia emocional los esfuerzos llevados a cabo hasta la fecha han consistido en adaptar los instrumentos ya existentes, y los resultados no han sido muy halagüeños, de ahí la necesidad del desarrollo y construcción de un instrumento específico para el deporte y que recoja las peculiaridades que estas actividades poseen.

\section{Bibliografía}

1. Arruza, J. A., Arribas, S., González, O, Balagué, G., Romero, S., y Ruiz, L. M. (2005). Desarrollo y validación de una versión preliminar de la escala de competencia emocional en el deporte (ECE-D). Revista Motricidad, 14, 153-163.

2. Austin, E. J., Saklofske, D.H., Huang, S. H. S., y McKenney, D. (2004). Measurement of trait emotional intelligence: Testing and cross-validating a modified version of Schutte et al. (1998) measure. Personality and Individual Differences, 36, 555-562.

3. Bar-On, R. (1996). The Emotional Quotient Inventory (EQ-i): A test of emotional intelligence. Toronto, Canada: Multi Health Systems, Inc.

4. Byrne, B. M. (2006). Structural equation modeling with EQS. Mahwah, NJ: LEA Publishers.

5. Chan, J.T. y Mallett, C.J. (2011) The value of Emotional Intelligence for high performance coaching. International Journal of Sports Science o Coaching, 6, 3, 315-328.

6. Chico, E. (1999). Evaluación psicométrica de una escala de inteligencia emocional. Boletín de Psicología, 62, 65-78.

7. Ciarrochi, J., Deane, F. P., y Anderson, S. (2002). Emotional intelligence moderates the relationship between stress and mental health. Personality and Individual Differences, 32, 2, 197-209.

8. Côte, S., y Miners, C. (2006). Emotional intelligence, Cognitive intelligence and Job Performance. Administrative Science Quarterly, 51, 1-28.

9. Crombie, D., Lombard, C. y Noakes, T. (2009). Emotiona Intelligence scores predict team sports performance in National Cricket competition. International Journal of Sport Science \& Coaching, 4, 2, 209 - 224.

10. DeVellis, R. F. (2003) Scale development: Theory and applications (2a Ed.). Thousand Oaks, CA: Sage.

11. Extremera, N., Fernández-Berrocal, P., Mestre, J. M., y Guil, R. (2004). Medidas de evaluación de la inteligencia emocional. Revista Latinoamericana de Psicología, 36, 209-228.

12. Extremera, N., y Fernández-Berrocal, P. (2004). El uso de las medidas de habilidad en el ámbito de la inteligencia emocional. Ventajas e inconvenientes con respecto a las medidas de auto-informe. Boletín de Psicología, 80, 59-77.

13. Ferrandiz, C., Martín, F., Gallud, L., Ferrando, M., López Pina, J. A., y Prieto, M. D. (2006). Validez de la escala de inteligencia emocional de Schutte en una muestra de estudiantes universitarios. Ansiedad y Estrés, $12,167-179$.

14. Gardner, H. (1983). Frames of mind: The theory of multiple intelligences. New Cork: Basic Books.

15. Goleman, D. (1996). Inteligencia emocional. Barcelona: Kairós.
16. Goleman, D. (1998). La práctica de la inteligencia emocional. Barcelona: Kairós.

17. González, O. (2008). Análisis y validación de un cuestionario de inteligencia emocional en diferentes contextos deportivos. Tesis Doctoral son publicar, Universidad del País Vasco, San Sebastián. Espańa

18. Graupera, J. L. (2007). Estilos de aprendizaje en la actividad física y el deporte. Tesis Doctoral sin publicar, Universidad de Castilla-La Mancha, Toledo. Espańa

19. Hanin, Y.L. (2000). Emotion in sports. Champaign. Human kinetics.

20. Lane, A.M., Devonport, T.J., Soos, I., Karsai, I, Leibinger, E. \& Hamar, P. (2010). Emotional intelligence and emotions associated with optimal and dysfunctional athletic performance. Journal of Sports Science and Medicines, 9, $388-392$.

21. Lane, A., Meyer, B., Devonport, T., Davies, K., Thelwell, R., Gill, G. Diehl, C., Wilson, M., y Weston, N. (2009). Validity of the emotional intelligence scale for use in sport. Journal of Sports Science and Medicine, 8, 289-295.

22. Lane, A.M., Thelwell, R.C., Lowther, J. y Devonport, T.J. (2009) Emotional intelligence and psychological skills use among athletes. Social Behavior and Personality, 37, 2, 195-202.

23. Marsh, H. W. (2007). Application of confirmatory factor analysis and structural equation modeling in sport and exercise psychology. En G. Tenenbaum y R. C. Eklund (Eds.), Handbook of on sport psychology (3rd ed., pp. 774 - 798). New York: Wiley.

24. Matthews, G., Zeidner, M., y Roberts, R. D. (2002). Emotional intelligence: Science and myth. Cambridge, MA: MIT Press.

25. Mayer, J. D., y Salovey, P (1993). The intelligence of emotional intelligence. Intelligence, 17, 433-442.

26. Mayer, J. D., y Salovey, P (1997). What is emotional intelligence? En P. Salovey y D. Sluyter (Eds), Emotional development and emotional intelligence: Educational implications (pp. 3-31). New York: Basic Books.

27. Meyer, B., y Zizzi, S. (2007). Emotional Intelligence in sport: conceptual, methodological, and applied issues. En A. M. Lane (Eds.), Mood and human performance conceptual, measurement and applied issues (pp. 131-152). Nova Science Publishers: Houppauge, N.Y.

28. Meyer, B. B., y Fletcher, T. B. (2007). Emotional intelligence: A theoretical overview and implications for research and professional practice in sport psychology. Journal of Applied Sport Psychology, 19, 1 - 15.

29. Netemeyer, R. G., Bearden, W. O., y Sharma, S. (2003) Scaling procedures: Issues and applications. Thousand Oaks, CA: Sage.

30. Nunnally, J.C. (1978). Psycometric theory. Nueva York: McGraw-Hill. 
31. Pérez, C. (2004). Técnicas de análisis multivariante de datos. Madrid: Pearson-Prentice Hall.

32. Pérez, N., y Castejón, J. L. (2007). La inteligencia emocional como predictor del rendimiento académico en estudiantes universitarios. $A n-$ siedad y Estrés, 13, 121-131.

33. Petrides, K. V., y Furnham, A. (2000). On the dimensional structure of emotional intelligence. Personality and Individual Differences, 29, 313320.

34. Prieto, M. D., Ferrándiz, C., Sánchez, C., y Bermejo, R. (2008). Inteligencia emocional y alta habilidad. Revista Española de Pedagogía, 240, 241-260.

35. Saklofske, D. H., Austin, E. J., y Minski, P. S. (2003). Factor structure validity of a trait emotional intelligence measure. Personality and Individual Differences, 34, 707-721.

36. Salovey, P., y Mayer, J. D. (1990). Emotional intelligence. Imagination, Cognition and Personality, 9,185-211.

37. Salovey, P., Hsee, C., y Mayer, J. D. (1993). Emotional intelligence and the regulation of affect. En D. M. Wegner, y J. W. Pennebaker (Eds.), Handbook of mental control (pp. 258-277). Englewood Cliffs, NJ: Prentice Hall.

38. Schutte, N. S., Malouff, J. M., Hall, L. E., Haggerty, D. J., Cooper, J. T., Golden, Ch. J., y Dornhein, L. (1998). Development and validation of a measure of emotional intelligence. Personality and Individual Differences, $25,(2), 167-177$.

39. Thelwell, R. C., Lane, A. M., Wetson, N. J. V., y Greenlees, I. A. (2008). Exmining relationships between emotional intelligence and coaching efficacy. International Journal of Sport and Exercise Psychology, 6, 224235.

40. Zeidner, M., Matthews, G., y Roberts, R. D. (2004). Emotional intelligence in the workplace: a critical review. Applied Psychology: An International Review, 53, 371-399.

41. Zizzi, S. J., Deaner, H. R., y Hirschhorn, D. K. (2003). The relationship between emotional intelligence and performance among college baseball players. Journal of Applied Sport Psychology, 15, 262-269. 


\section{Apéndice 1. \\ Inventario de Inteligencia Emocional adaptado al Deporte (IED) (adaptación de Ruiz, García y Graupera)}

\section{Instrucciones:}

Conteste a continuación al listado de frases relacionadas con la forma en que se siente en si deporte. Le solicitamos que de manera sincera indique el acuerdo o desacuerdo que mantiene con las mismas, para lo cual rodeará con un círculo el número que mejor recoja si opinión, recordando que el 1 significa que está en total desacuerdo y el 5 en total acuerdo.

Gracias por su colaboración

1. Sé cuando tengo que hablar de mis problemas personales a otros/as compañeros/as del equipo.

2. Cuando me encuentro ante problemas, recuerdo las veces que me enfrenté a otros problemas similares y cómo los superé.

3. En la mayoría de las cosas que intento espero hacerlas bien.

4. Otros/as compañeros/as encuentran fácil el poder confiar en mí.

5. Sé como se encuentran otros compañeros/as solo escuchando el tono de su voz.

6. Algunos de los acontecimiento más relevantes de mi vida me han provocado que vuelva a pensar qué es lo importante y lo qué no lo es.

7. Cuando mi estado de ánimo cambia, veo nuevas posibilidades de juego.

8. Las emociones son una de esas cosas de la vida que me hacen sentir valioso/a.

9. Soy consciente de mis emociones tal y como las vivo en el juego o competición.

10. Espero que ocurran cosas buenas.

11. Me gusta compartir mis emociones con mis compañeros/as de equipo.

12. Cuando experimento una emoción positiva, sé como hacer que esto dure.
13. Arreglo las cosas para que otros se diviertan.

14. Busco cosas en el deporte que me hagan sentir bien.

15. Soy consciente de los mensajes no verbales que envío a los/as otros/as compañeros/as.

16. Me presento ante los demás de tal manera que les de buena impresión.

17. Cuando estoy en un buen estado de ánimo, me resulta fácil resolver los problemas del juego.

18. Viendo las expresiones faciales de los demás, sé las expresiones que están experimentando.

19. Sé por qué cambian mis emociones.

20. Cuando estoy en buen estado de ánimo. Soy capaz de generar buenas ideas en el juego.

21. Tengo control sobre mis emociones en el entrenamiento y/o competición.

22. Reconozco fácilmente las emociones tal y como las experimento.

23. Me motivo a mí mismo/a imaginándome un buen resultado en las tareas que llevo a cabo.

24. Felicito a los demás cuando hacen las cosas bien al competir.

25. Soy consciente de los mensajes no verbales que me envían otras personas.

26. Cuando otra persona me cuenta algún acontecimiento importante de su vida, lo siento como si yo también lo hubiera experimentado.

27. Cuando siento un cambio en mis emociones, tiendo a tener nuevas ideas.

28. Acostumbro a tener buen ánimo para ayudarme a persistir ante los obstáculos.

29. Con solo mirarles, sé lo que otros/as compañeros/as o contrincantes están sintiendo.

30. Ayudo a otros/as compañeros/as a sentirse mejor cuando están en momentos bajos. 